\title{
Proposta metodológica para o módulo de consumo alimentar pessoal na pesquisa brasileira de orçamentos familiares
}

\author{
Methodological proposal for the individual food \\ intake module of the Brazilian household budget survey
}

\author{
Edna Massae YOKOO' \\ Rosângela Alves PEREIRA² \\ Glória Valéria da VEIGA² \\ Siléia NASCIMENTO2 \\ Rosana Salles COSTA \\ Vânia Maria RAMOS DE MARINS ${ }^{3}$ \\ Jackeline Christiane Pinto LOBATO ${ }^{4}$ \\ Rosely SICHIERI ${ }^{4}$
}

\section{RES U M O}

As alterações que vêm ocorrendo no cenário epidemiológico brasileiro, nas últimas décadas, resultam de profundas modificações sociais e econômicas que incluem, entre outros aspectos, mudanças nos hábitos de alimentação. No Brasil, o único estudo que investigou o consumo alimentar da família com abrangência nacional foi o Estudo Nacional sobre Despesa Familiar, em 1974-1975. Embora sejam de utilidade incontestável, estudos nacionais de consumo alimentar são onerosos e poucos países conseguem desenvolvê-los regularmente. Por outro lado, as pesquisas de orçamentos familiares são importantes fontes de dados de disponibilidade de alimentos no domicílio, a qual é inferida a partir do registro da compra de produtos alimentícios. As recentes modificações nos hábitos de consumo, particularmente, a realização de refeições fora do domicílio constituem limitações para a utilização de dados das pesquisas brasileira de orçamentos familiares como estimativa do consumo alimentar. Assim, o governo brasileiro propôs que a próxima pesquisa de orçamento familiar, a ser desenvolvida em 2008-2009, incluísse um módulo de consumo alimentar individual. As informações sobre o consumo dietético individual serão utilizadas para completar os dados sobre compra de alimentos. O objetivo

\footnotetext{
1 Universidade Federal Fluminense, Instituto de Saúde da Comunidade, Departamento de Epidemiologia e Bioestatística. R. Marques de Paraná, 303, HUAP, $3^{\circ}$ andar, Prédio Anexo, 24030-210, Centro, Niterói, RJ, Brasil. Correspondência para/Correspondence to: E.M. YOKOO.E-mail: <eyokoo@terra.com.br>.

2 Universidade Federal do Rio de Janeiro, Centro de Ciências da Saúde, Instituto de Nutrição Josué de Castro. Rio de Janeiro, RJ, Brasil.

3 Fundação Arthur Sá Earp, Faculdade de Nutrição. Petrópolis, RJ, Brasil.

4 Universidade Estadual do Rio de Janeiro, Instituto de Medicina Social, Departamento de Medicina Social. Rio de Janeiro, RJ, Brasil.
}

Financiamento: Ministério da Saúde (convênio no 4652/2005). 
deste trabalho é relatar a construção e o desenvolvimento da metodologia a ser utilizada no módulo de consumo alimentar individual da pesquisa de orçamento familiar 2008-2009. Os dados a serem obtidos serão combinados com as informações de disponibilidade de alimentos no domićlio para estimar o consumo alimentar usual individual.

Termos de indexação: Consumo alimentar. Epidemiologia nutricional. Hábitos alimentares. Inquéritos alimentares. Orçamentos.

\section{A B S T R A C T}

The changes that have been happening in the Brazilian epidemiological scenario in the last decades result from deep social and economic modifications that include, among other things, changes in dietary habits. In Brazil, the only study that investigated family food consumption nationally was the National Family Expenditure Study done from 1974 to 1975. Although useful, national food consumption studies are expensive and only a few countries can conduct them regularly. Nonetheless, household budget surveys are important sources of data on the availability of food at home determined by records of foods purchased. Recent changes in consumption habits, particularly eating out, limit the use of data from household budget surveys to estimate food intake. Thus, the Brazilian government suggested that the next household budget survey to be done in 2008-2009 include a module on individual food consumption. Information on individual food intake will be used to supplement the data regarding food purchases. The objective of this study is to report the development of the methodology to be used in the module of individual food consumption of the household budget survey of 2008-2009. Budget data will be combined with intake data to estimate the usual individual food consumption.

Indexing terms: Food consumption. Nutritional epidemiology. Food habits. Diet surveys. Budgets.

\section{N T R O D U Ç Ã O}

O monitoramento do consumo alimentar e o reconhecimento das mudanças nos padrões de consumo de uma população são objeto das ciências da Nutrição em estudos epidemiológicos, e representam contribuições para o aprimoramento das ações de saúde. Esse conhecimento tem grande valor para a fundamentação de guias e recomendações nutricionais, para o planejamento de políticas agrícolas, de alimentação e nutrição, de prevenção e controle de enfermidades, de regulamentação da aplicação de pesticidas e de incorporação de vitaminas, minerais e aditivos em alimentos industrializados, além de terem relevância para as investigações etiológicas.

O desenvolvimento agrícola nas últimas décadas levou ao aumento da disponibilidade de alimentos e ao incremento da produção de alimentos industrializados. Por outro lado, a crescente urbanização da população e o acesso a produtos processados acarretaram modificações na alimentação de grande parcela da população.
No Brasil, a disponibilidade de alimentos cresce associada a mudanças na composição da dieta, principalmente no aumento da disponibilidade de produtos como carnes, leites e derivados, óleos e gorduras vegetais, biscoitos e refrigerantes e redução de cereais e leguminosas ${ }^{1}$.

As alterações na alimentação são parte das profundas mudanças sociais e econômicas que determinam, em última instância, alterações no cenário sanitário, particularmente quanto ao padrão de morbimortalidade e de nutrição e alimentação. Nesse quadro, observa-se que a desnutrição, embora declinante, está ainda presente de forma significativa particularmente em segmentos sociais menos privilegiados, como as populações das periferias dos grandes centros urbanos ${ }^{2,3}$. Por outro lado, o sobrepeso e a obesidade apresentam incremento generalizado, em todas as regiões, grupos sociais e etários, o que vem sendo acompanhado do aumento das prevalências de diabetes tipo 2 e dislipidemias ${ }^{4,5}$.

Dados padronizados por idade de pesquisas nacionais desenvolvidas pelo Instituto Brasileiro de 
Geografia e Estatística (IBGE) em 1974-1975, em 1989 e em 2002-2003 mostram que a prevalência do baixo peso em adultos reduziu-se em 50,0\% entre a primeira e a segunda investigação. Em contrapartida, a prevalência do excesso de peso e da obesidade aumentou de forma contínua e intensa. Entre os homens, a prevalência de obesidade triplicou no período de 30 anos que separa a primeira e a última pesquisa, passando de 2,8\% para $8,8 \%$. Já entre as mulheres, a evolução do excesso de peso e da obesidade se dá de forma diferenciada: ocorreram aumentos da ordem de 50,0\% entre 1974-1975 e 1989, e a estabilização dessas taxas entre 1989 e 2002-2003 em patamares elevados: neste último inquérito, 39,7\% das mulheres foram classificadas com sobrepeso e $12,7 \%$ com obesidade ${ }^{6}$.

Uma vez que hábitos alimentares inadequados são um dos fatores ambientais que podem estar mais diretamente ligados a este aumento na prevalência de obesidade, a investigação do consumo alimentar da população brasileira pode trazer grande contribuição para melhor compreensão sobre a gênese do problema. No Brasil7, o único estudo que investigou o consumo alimentar da família com abrangência nacional foi o Estudo Nacional sobre Despesa Familiar (ENDEF), realizado pelo IBGE, em 1974-1975. Nesse estudo o consumo alimentar de uma amostra de, aproximadamente, 55 mil famílias foi investigado por meio de pesagem direta, sendo também relatados os alimentos que foram consumidos fora do domicílio durante todo o período. A partir de informações sobre o consumo global da família, a ingestão diária de energia e nutrientes de cada membro da família foi estimada considerando dados de sexo, idade e a participação nas refeições. O estudo revelou que mais de $2 / 3$ das famílias apresentavam consumo de energia abaixo do recomendado e que o déficit médio de aporte energético era em torno de $400 \mathrm{kcal}^{7}$.

Em meados da década de 1990, uma iniciativa do extinto Instituto Nacional de Alimentação e Nutrição (INAN) levou ao desenvolvimento de um estudo multicêntrico que levantou infor- mações sobre aquisição de alimentos em cinco municípios (Campinas, Rio de Janeiro, Ouro Preto, Goiânia e Curitiba) e sobre consumo alimentar no Rio de Janeiro, onde foi aplicado um questionário de freqüência de consumo de alimentos ${ }^{6}$. Esse estudo detectou inadequação no consumo energético nas famílias com renda per capita de até um salário-mínimo. Enquanto que famílias com renda global de até dois salários-mínimos apresentavam risco de consumo inadequado de ferro, cálcio e retinol. Os dados mostraram que a proporção da energia fornecida por gorduras mantinha-se dentro do limite recomendado de $30 \%$; entretanto, o consumo de gordura saturada e colesterol estava acima do recomendado para parcelas expressivas da população: o consumo de colesterol maior do que 300mg por dia foi observado em $58 \%$ dos homens e $43 \%$ das mulheres do Rio de Janeiro; enquanto que em Goiânia, 44\% dos homens e $51 \%$ das mulheres apresentaram consumo de colesterol acima do limite recomendado ${ }^{8}$.

Embora sejam de utilidade incontestável, estudos nacionais de consumo alimentar são onerosos e poucos países conseguem desenvolvêlos regularmente. Por outro lado, as pesquisas de orçamentos familiares (POF) são importantes fontes de dados de disponibilidade de alimentos no domicílio, a qual é inferida a partir do registro da compra de produtos alimentícios. São inquéritos desenhados para, de um modo geral, atender às demandas do setor econômico. Uma das vantagens desses inquéritos é o fato de que são desenvolvidos a intervalos regulares desde a década de 1980 em diversos países com metodologia similar, abrangendo amostras representativas da população de cada país 9 . Contudo, as pesquisas de orçamentos familiares não possibilitam reconhecer o consumo individual de alimentos propriamente dito.

No Brasil, O IBGE desenvolveu POF em 1986-1987, 1995-1996 e em 2002-2003. A POF brasileira "visa mensurar, fundamentalmente, as estruturas de consumo, dos gastos e dos rendimentos das famílias e possibilita traçar um perfil 
das condições de vida da população brasileira a partir da análise de seus orçamentos domésticos" ${ }^{\prime \prime}$. Os dados das POFs foram comparados aos dados de 1974-1975 do ENDEF para análises sobre a evolução dos indicadores da disponibilidade alimentar no Brasil, entretanto, segundo o próprio IBGE essas análises podem ser consideradas "uma estimativa conservadora das mudanças que devem ter ocorrido no padrão da dieta brasileira nas últimas três décadas" ${ }^{\prime}$.

A mais recente POF, realizada em 2002-2003, coletou informações sobre alimentos comprados, produzidos e recebidos como doação ou presente para estimar a disponibilidade de alimentos de cerca de 50 mil domicílios de todas as regiões do País. Essa pesquisa também incluiu dados de despesas com alimentos consumidos fora de casa. As informações sobre despesas com alimentos em cada domicílio possibilitam a estimativa da disponibilidade de alimentos e de energia e nutrientes per capita por ano'.

As recentes modificações nos hábitos de consumo, particularmente, a realização de refeições fora do domicílio, constituem limitações para a utilização de dados das POFs como estimativa do consumo alimentar. Na POF de 2002-2003, cada indivíduo do domicílio selecionado anotou, pelo período de uma semana, suas despesas com refeições prontas fora do domicílio. A coleta desses dados mostrou que seria viável o levantamento de dados sobre consumo efetivo individual acoplado à POF. Assim, o governo brasileiro propôs que a próxima POF, a ser desenvolvida em 2008-2009, incluísse um módulo de consumo alimentar individual.

As informações sobre o consumo dietético individual serão utilizadas para completar os dados sobre compra de alimentos. A combinação das duas metodologias de coleta de dados é uma forma de reduzir custos, além de aproveitar a larga experiência do IBGE nesse tipo de investigação.

O desenvolvimento de um instrumento para avaliar o consumo individual teve início em setembro de 2005, quando um fórum de especialistas foi convocado pelo Ministério da Saúde. A idéia era adotar um instrumento que permitisse não somente reconhecer o padrão de consumo alimentar e avaliar o consumo de energia, ferro, vitamina $A$, acido fólico, entre outros nutrientes.

O objetivo deste trabalho é relatar a construção e o desenvolvimento da metodologia a ser utilizada no módulo de consumo alimentar individual da POF 2008-2009. Os dados a serem obtidos serão combinados com as informações de disponibilidade de alimentos no domicílio para estimar o consumo alimentar usual individual.

\section{M É T O D O S}

\section{Desenho da amostra e dados de disponibilidade de alimentos no domicílio}

O desenho da amostra será estruturado de modo a propiciar a apresentação de resultados para o Brasil e para as Grandes Regiões (Norte, Nordeste, Sudeste, Sul e Centro-Oeste), segundo a situação urbana ou rural. Entretanto, para as unidades da federação, as nove regiões metropolitanas (Belém, Fortaleza, Recife, Salvador, Belo Horizonte, Rio de Janeiro, São Paulo, Curitiba, Porto Alegre) e as capitais dos estados os resultados se referirão apenas às populações urbanas.

A obtenção dos dados de aquisição de alimentos manterá o mesmo formato adotado na POF de 2002-2003, que coletou informações sobre disponibilidade de alimentos e bebidas no domicílio, considerando compra e aquisições não monetárias, como doações, produção, trocas etc. Para a obtenção dessas informações será utilizada a "Caderneta de Despesa Coletiva", na qual o informante, em geral, a pessoa responsável por essas despesas no domicílio, anotará durante sete dias consecutivos a descrição dos produtos adquiridos, suas quantidades, o local, a forma de aquisição e o valor dispendido. 


\section{O módulo de consumo alimentar individual na POF 2008-2009}

Para o planejamento desse módulo, o Ministério da Saúde congregou pesquisadores de todas as regiões do País (Anexo 1), que definiram o processo de coleta de dados, incluindo o manual de coleta de dados, o processo de entrada dos dados no programa específico do IBGE, o treinamento dos agentes de pesquisa do IBGE e a elaboração de orientações para os entrevistados anotarem o seu consumo alimentar. Este módulo será o questionário POF 7 - Bloco de Consumo Alimentar Pessoal. Em linhas gerais, a obtenção de dados de consumo alimentar na POF 2008-2009 terá como principais elementos metodológicos os descritos a seguir.

\section{População de estudo}

Os dados serão coletados em uma subamostra correspondente a $25 \%$ de todas as famílias investigadas, e serão obtidos dados de todos os indivíduos com pelo menos 10 anos de idade nos domicílios selecionados. Estima-se que serão selecionados 20 mil domićlios, dos 80 mil previstos para compor a amostra da POF. Tendo por base que a média de indivíduos com idades acima de 10 anos é de 3,5 pessoas em cada domicílio, deverão ser coletados dados de, aproximadamente, 70 mil indivíduos.

Para as crianças menores de 10 anos haverá um grupo de perguntas sobre a realização de refeições e o local onde estas são usualmente realizadas. Um adulto deverá fornecer essa informação.

Gestantes e nutrizes preencherão os registros, mas seus dados não serão incluídos na estimativa do consumo individual, devido ao fato de que são esperadas modificações no consumo alimentar durante essas fases, além de ocorrer mudanças nas necessidades nutricionais. Por outro lado, gestantes e nutrizes constituem um grupo muito restrito para que seus dados sejam usados para estimar o seu consumo específico. Porém, as informações dessas mulheres serão usadas para comparar os dados de consumo individual com os de compra de alimentos da família.

\section{Método de obtenção de dados de consumo de alimentos}

Um critério primordial na escolha do método para avaliar o consumo alimentar, é estabelecer se o que se quer avaliar é o consumo usual (habitual) ou o atual. O consumo usual está relacionado aos hábitos alimentares praticados em um período de tempo determinado (um ano, um semestre etc), enquanto que o consumo atual representa o consumo alimentar em um ponto do tempo (um, dois ou mais dias).

Em pesquisas epidemiológicas os métodos mais utilizados para obter dados sobre consumo alimentar são: a) folha de balanço; b) inventário; c) pesquisa de orçamento familiar (POF); d) registro ou diário alimentar; e) métodos recordatórios; f) questionário de freqüência alimentar (QFA). Os itens $a, b$, e c permitem avaliações nos agregados como: países, famílias, grupos sociais e os demais podem avaliar tanto o consumo dos agregados quanto dos individuais, com a ressalva de que a aplicação de um único registro ou recordatório de 24 horas, em geral, é adequado para avaliação de agregados e não de indivíduos ${ }^{10}$.

A escolha do método de registro de alimentos consumidos em dois dias para a POF 2008-2009 deveu-se à necessidade de interferir o mínimo possível na prática de coleta de dados em campo desenvolvida pelo IBGE, que vem aplicando para coleta de informações o registro de despesas em uma caderneta.

O método de registro alimentar apresenta algumas vantagens, como o fato de não depender da memória, uma vez que os alimentos são anotados no momento do consumo e, usualmente, há maior precisão e exatidão na informação quanto às porções ingeridas. Por outro lado, este método apresenta algumas limitações, pois o consumo pode ser alterado em decorrência de o indivíduo 
saber que está sendo avaliado; assim, a qualidade do registro depende muito da colaboração do entrevistado e necessita de habilidades de escrita para a sua realização'11.

A validade do método a ser empregado na POF 2008-2009 será avaliada segundo o método da água duplamente marcada ${ }^{12}$, considerando dados coletados em 100 indivíduos adultos do Município de Duque de Caxias, Rio de Janeiro.

\section{Coleta de dados}

Aos entrevistados será solicitado que completem dois registros alimentares (POF 7), considerando dias não consecutivos, que deverão incluir os itens alimentares (alimentos ou preparações), a quantidade consumida (em medidas caseiras ou em medidas de volume), o horário e o local de consumo (no domicílio - que inclui todos alimentos preparados no domicílio, mesmo que levados para consumir em outro lugar, e fora do domicílio). Haverá uma pergunta à parte sobre o consumo de açúcar e adoçante. Não será solicitado o registro do consumo de água.

O Bloco de Consumo de Alimentar Pessoal (POF 7) para anotação do consumo alimentar contém três páginas: a primeira tem um exemplo de como anotar; a segunda está em branco para o indivíduo anotar tudo o que comer no primeiro dia e a terceira está em branco para anotar o que comer no segundo dia não consecutivo.

O agente de pesquisa do IBGE revisará as informações registradas junto com o informante no momento de digitar os dados no programa apropriado. Nesse momento o agente de pesquisa investigará se houve omissão de itens, refinará a informação sobre quantidades consumidas, elucidará situações que possam dar margem à confusão como preparações ou alimentos não reconhecidos, longos períodos sem registro de consumo ou poucos itens (menos de cinco) registrados para um dia.
Ainda nessa oportunidade, o informante deverá ser indagado sobre o consumo de itens que, usualmente, são omitidos em registros alimentares como (a) manteiga, margarina, geléia, mel, melado, ou outros produtos colocados em pães, biscoitos, bolos, batata-doce, aipim (macaxeira, mandioca), batata etc.; (b) bebidas: café, chá, sucos, refrigerantes, bebidas alcoólicas etc. (c) pequenos lanches, consumidos ao longo do dia tais como: salgados, salgadinhos, bolachas, balas, chicletes, doces etc. Além disso, o agente de pesquisa deverá verificar se os produtos consumidos são dietéticos ou com redução de valor energético. Para itens específicos será solicitado o detalhamento da forma de preparação (frito, cozido, assado etc.).

\section{Entrada dos dados de consumo alimentar em banco de dados computadorizado}

Para viabilizar a entrada dos dados de consumo alimentar em programa de computador apropriado foi criada uma base de dados que inclui alimentos, preparações, modo de preparo e unidades de medida. A lista de alimentos baseou-se nos 5.686 itens registrados nas POFs anteriores. Entretanto, grande parte desses registros é relativa a sinonímias (por exemplo: mandioca, macaxeira, aipim) ou variedades (por exemplo, laranja-pêra, laranja-lima etc.) ou contempla grafias não usuais (jiló, "giló"). Para alguns itens foram mantidas apenas as designações genéricas: por exemplo, de cerca de 2 mil termos utilizados para identificar diferentes variedades de peixe foram mantidos: "peixe inteiro de água doce", "peixe inteiro de mar", "peixe inteiro não especificado", "peixe salgado de água doce", "peixe salgado de mar", "peixe salgado não especificado".

Para dar suporte à digitação do consumo relatado, na preparação da base de dados de alimentos, foram respeitadas as nomenclaturas regionais e a diversidade do consumo alimentar das diferentes regiões do País. A lista de alimentos também inclui as preparações mais tradicionais e 
populares como, por exemplo, feijoada, lasanha, vatapá, moqueca, macarronada, salada crua, salada cozida, entre outros.

As informações registradas nos dois bancos de dados de alimentos, o de compra e o de consumo, serão cruzadas; com isso será possível complementar as informações obtidas na pesquisa de consumo individual efetivo.

A entrada de dados ocorrerá, sempre que possível, em computadores portáteis nos domicílios durante a revisão do registro. Os agentes de pesquisa poderão incluir novos itens, mas durante o treinamento e no manual serão intensivamente recomendados a fazê-lo somente quando não puderem encontrar um item que descreva o alimento ou a preparação relatada.

Cada item nos registros individuais será relacionado às porções relatadas em medidas de volume (gramas, mililitros etc.) ou unidades de medidas já padronizadas (xícaras, copos, colheres, pratos, pegadores, garrafas, latas etc.). Os agentes de pesquisa não poderão incluir novas unidades de medida na base de dados e serão instruídos a procurarem identificar a unidade de medida que melhor descreve aquela reportada pelo informante. Para padronizar o porcionamento das quantidades relatadas os agentes de pesquisa serão treinados na identificação das medidas caseiras indicadas na base de dados, como diferentes tipos de talheres, copos, pratos, garrafas etc.

Para itens específicos, como carnes e peixes, o programa de entrada de dados solicitará que o agente de pesquisa registre a forma de preparação: cozido, frito, assado, empanado etc. Esses procedimentos garantirão a padronização da entrada de dados e a qualidade do relato do consumo individual de alimentos.

\section{Viabilização do trabalho de campo}

Para o trabalho de campo foram elaborados dois manuais: um para o entrevistador (agente do IBGE) e outro para o entrevistado.
O primeiro manual de instruções tem como objetivo ajudar o entrevistador do IBGE a orientar o entrevistado sobre como preencher o POF 7, com os detalhes sobre os alimentos, consumo fora ou dentro do domicilio, as medidas caseiras a partir da lista estabelecida, exemplos de POF 7 preenchido, e orientações para a revisão do registro.

O segundo manual é uma cartilha direcionada aos entrevistados, com o objetivo de apresentar a POF 7 e orientar, de forma resumida, o preenchimento da caderneta de consumo.

Constará do manual do entrevistador e das orientações aos entrevistados um conjunto de fotografias com medidas caseiras para facilitar o registro das quantidades consumidas.

Para o treinamento dos agentes de pesquisa foram desenvolvidos apresentações em meio digital e exercícios práticos. Esse treinamento será repetido em cada agência do IBGE pelos supervisores e coordenadores estaduais. Estes últimos, por sua vez, foram treinados por técnicos do IBGE e da equipe de elaboração do módulo POF 7.

Após a elaboração do instrumento de coleta, para viabilização do trabalho, no ano de 2007, foi realizado um estudo piloto pelo IBGE, em duas cidades, uma no estado de Santa Catarina, onde foram entrevistados 19 domicílios e outra em Alagoas, onde 27 famílias foram entrevistadas, totalizando 142 indivíduos no relato de aquisição de alimentos e 40 pessoas no registro da POF 7 . A partir desse estudo piloto, foram realizadas modificações no instrumento de coleta de dados e nos manuais de instrução.

No segundo semestre de 2007, após refinamento dos protocolos pela equipe de pesquisadores, estes foram novamente testados em dois momentos. No primeiro foram aplicados em 300 indivíduos adultos do município de Duque de Caxias, Estado do Rio de Janeiro. Após adaptações do instrumento com base nessa simulação, foi realizada uma segunda simulação tendo estudantes de uma universidade pública do Rio de Janeiro da área de humanas e tecnológica como entrevistadores e entrevistados. Nessa etapa, os 
estudantes entrevistadores aplicaram a POF 7, conforme treinamento recebido. Após estas duas etapas, o manual de treinamento do entrevistador e a cartilha para o entrevistado, assim como os instrumentos digitais, passaram por ampla revisão pela equipe de pesquisadores até atingirem o formato final para o treinamento dos supervisores de trabalho de campo do IBGE.

\section{RES U LTA DOS}

O plano de análise será desenvolvido, em separado, para famílias com e sem crianças menores de 10 anos. Além de considerar o desenho amostral complexo, a análise de dados deverá contemplar os seguintes aspectos:

1) Constituição da base de dados de composição dos alimentos incluídos no programa de entrada dos dados e daqueles adicionados pelos agentes de pesquisa. Para tanto serão utilizados dados de tabelas de composição de alimentos disponíveis no Brasil, com prioridade para a tabela da $\mathrm{TACO}^{13}$ e a tabela do IBGE ${ }^{14}$.

2) Estimativa do consumo médio per capita semanal de alimentos, grupos de alimentos, energia e nutrientes. Para esta estimativa serão considerados os dados dos dois registros e a estimativa será corrigida pelo fator de deatenuação $(\lambda)$ que é dado pela razão entre a variância entre-indivíduos/variância intra-individual ${ }^{15}$, como apresentado na equação 1 .

$\begin{array}{l}\text { consumo semanal } \\ \text { per capita }\end{array}=\left[\left(\frac{\text { registro } 1+\text { registro } 2}{2}\right) * 7\right] * \lambda$ (equação 1$)$

na qual:

$\lambda=\frac{S_{w}^{2}}{S_{b}^{2}} ; S_{w}^{2}=$ variância intra-individual; $S_{b}^{2}=$ variância entre-indivíduos

3) Estimativa da disponibilidade domiciliar de alimentos, grupos de alimentos, energia e nutrientes: será dada a partir das informações sobre aquisição de alimentos. As aquisições de cada domicílio serão divididas pelo número de moradores para estimar a disponibilidade per capita, a qual será posteriormente comparada com as estimativas de consumo per capita.

4) Parâmetros a serem estimados: será estimado o consumo diário médio per capita de grupos de alimentos, energia e nutrientes. Para a caracterização do consumo alimentar da população brasileira os dados serão agregados segundo a localização (grandes regiões, estados, capitais, regiões metropolitanas), situação urbano/rural, categorias de renda, escolaridade do chefe da família e composição familiar.

5) Comparação dos dados de consumo individual e de aquisição familiar de alimentos: a comparação de ambos os inquéritos permitirá estabelecer a validade de utilizar informações de pesquisas de orçamento familiar como substitutos de informações sobre consumo usual. Também será possível avaliar questões como sub-relato em inquéritos alimentares que utilizam o registro e dimensionar o efeito da realização de refeições fora do domicílio no consumo alimentar da população.

\section{CONSIDERAÇÕ ES FINAIS}

O módulo de consumo individual da POF contribuirá para o reconhecimento da situação de saúde e nutrição do País, juntamente com as informações de medidas antropométricas, além disso, o desenvolvimento de análise dos dados de consumo alimentar individual, combinados com as informações de aquisição de alimentos a serem obtidos na Pesquisa de Orçamentos Familiares 2008-2009, possibilitará a consolidação de técnicas e modelos de análise de consumo alimentar.

\section{COLABORADORES}

Todos os autores participaram da concepção do método descrito no artigo, assim como do delineamento do artigo. E.M. YOKOO coordenou a redação do manuscrito que foi revisada pelos co-autores. R. SICHIERI é coordenadora do projeto. 


\section{A GRADECIMENTOS}

André Martins (IBGE); Denise Petrucci Gigante (UFPel); Nélida Schmid Fornés (UFG); Regina Mara Fisberg (FSP/USP); Renata Bertazzi Levy (SES/SPP); Taís de Souza Lopes (INJC/UFRJ); Marília Mendonça Leão (CGPAN-MS); Janine Coutinho (CGPAN-MS).

\section{REFERÊ NCIAS}

1. Levy-Costa RB, Sichieri R, Pontes NS, Monteiro CA. Disponibilidade domiciliar de alimentos no Brasil: distribuição e evolução (1974-2003). Rev Saúde Pública. 2005; 39(4):530-40.

2. Salles-Costa R, Costa RS, Sichieri R. Avaliação alimentar em Duque de Caxias: desenho de estudos e resultados preliminares. Estud Av. 2007; 21(60):135-42.

3. Ferreira HS, Florêncio TMTM, Fragoso MAC, Melo FP, Silva TG. Hipertensão, obesidade abdominal e baixa estatura: aspectos da transição nutricional em uma população favelada. Rev Nutr. 2005; 18(2):209-18.

4. Escoda MSQ. Para a crítica da transição nutricional. Cienc Saude Col. 2002; 7(2):219-26.

5. Pinheiro ARO, Freitas SFT, Corso ACT. Uma abordagem epidemiológica da obesidade. Rev Nutr. 2004; 17(4):523-33.

6. Instituto Brasileiro de Geografia e Estatística. Pesquisa de Orçamentos Familiares - 2002-2003: aquisição alimentar domiciliar per capita, Brasil e grandes regiões. Rio de Janeiro; 2004.

7. Instituto Brasileiro de Geografia e Estatística. Estudo Nacional da Despesa Familiar - ENDEF: dados preliminares - consumo alimentar - antropometria. Região I - Estado do Rio de Janeiro/Região III Paraná, Santa Catarina, Rio Grande do Sul. Rio de Janeiro: IBGE; 1977. [mimeo].

8. Galeazzi MAM, Domene SMA, Sichieri R. Estudo multicêntrico sobre consumo alimentar. Campinas: Unicamp; 1997. [mimeo].

9. Lagiou P, Trichopoulou A. DAFNE contributors. The DAFNE initiative: the methodology for assessing dietary patterns across Europe using household budget survey data. Public Health Nutr. 2001; 4(5B):1135-41

10. Pereira RA, Sichieri R. Métodos de avaliação do consumo de alimentos. In: Kac G, Sichieri R, Gigante DP, organizadores. Epidemiologia nutricional. Rio de Janeiro: Atheneu; 2007. p.181-200.

11. Fisberg R, Slater $B$, Marchiori DML, Martini LA. Inquéritos alimentares: métodos e bases científicas. São Paulo: Manole; 2005.

12. Trabulsi J, Schoeller DA. Evaluation of dietary assessment instruments against doubly labeled water, a biomarker of habitual energy intake. Am J Physiol, Endocrinol Metab. 2001; 281(5): E891-E899.

13. Núcleo de Estudos e Pesquisas em Alimentação. Universidade Estadual de Campinas. Tabela brasileira de composição de alimentos - TACO. Versão 2 - 2a. ed. Campinas: Unicamp; 2006.

14. Instituto Brasileiro de Geografia e Estatística. Tabela de composição química de alimentos. $4^{a}$ ed. Rio de Janeiro: IBGE; 1996.

15. Willett W. Nutritional Epidemiology. 2nd ed. New York: Oxford University Press; 1998.

Recebido em: 14/4/2008

Aprovado em: 19/9/2008 


\section{ANEXO 1 \\ PARTICIPANTES DAS DIFERENTES ETAPAS DO GRUPO DE TRABALHO PARA O PLANEJAMENTO \\ DO MÓDULO CONSUMO INDIVIDUAL NA POF 2008-2009}

Antonia Trichopoulou (University of Athens)

Aline Cristina Souza Lopes (Universidade Federal de Minas Gerais - UFMG)

Ana Beatriz Vasconcelos (Coordenação Geral da Política Nacional de Alimentação e Nutrição - CGPAN/MS)

Ana Marlúcia de Assis (Centro Colaborador em Alimentação e Nutrição - Região Nordeste II)

André Martins (Instituto Brasileiro de Geografia e Estatística - IBGE)

Bethsáida Schmitz (Universidade de Brasilia - UnB)

Daniele Mendonça (Faculdade de Nutriçao da Universidade Federal Fluminense)

Denise Petrucci Gigante (Universidade Federal de Pelotas - UFPel)

Edílson Nascimento da Silva (Insituto Brasileiro de Geografia e Estatística - IBGE)

Edna Massae Yokoo (Departamento de Epidemiologia e Bioestatística da Universidade Federal Fluminense)

Elyne Engstron (Escola Nacional de Saúde Pública/Centro Colaborador em Alimentação e Nutrição - Região

Sudeste)

Estelamaris Tronco Monego (Centro Colaborador em Alimentação e Nutrição - Região Centro-Oeste)

Glória Valéria da Veiga (Instituto de Nutrição Josué de Castro da Universidade Federal do Rio de Janeiro)

Ida Helena C. F. Menezes (Centro Colaborador em Alimentação e Nutrição - Região Centro-Oeste)

Jackeline Christiane Pinto Lobato (Instituto de Medicina Social da Universidade Estadual do Rio de Janeiro)

Janine Giuberti Coutinho (Coordenação Geral da Política Nacional da Alimentação e Nutrição do Ministério da Saúde).

Márcia Vitolo (Fundação Faculdade Federal de Ciências Médicas de Porto Alegre)

Maria Del Carmen Molina (Universidade Federal do Espirito Santo - UFES)

Marília Mendonça Leão (Coordenação Geral da Política Nacional de Alimentação e Nutrição - CGPAN/MS)

Marina K. Ito (Universidade de Brasília - UnB )

Margareth Naves (Universidade Federal de Goiás - UFG/Faculdade de Nutrição)

Mônica Maria Osório (Universidade Federal de Pernambuco/Departamento de Nutrição)

Muriel Gubert (Coordenação Geral da Política Nacional de Alimentação e Nutrição - CGPAN/MS)

Nedja Silva Santos (Centro Colaborador em Alimentação e Nutrição - Universidade Federal de Pernambuco)

Nélida Schmid Fornés (Universidade Federal de Goiás/Faculdade de Nutrição)

Nézio dos Santos Pontes (Insituto Brasileiro de Geografia e Estatística - IBGE)

Rafael Moreira (Centro Colaborador em Alimentação e Nutrição - Região Sul)

Regina Mara Fisberg (Faculdade de Saúde Pública da Universidade de São Paulo)

Renata Bertazzi Levy (Instituto de Saúde-Secretaria Estadual de Saúde/Faculdade de Saúde Pública da Universidade de São Paulo)

Rosa Maria Dias (Centro Colaborador em Alimentação e Nutrição - Região Norte)

Rosana Salles (Instituto de Nutrição Josué de Castro da Universidade Federal do Rio de Janeiro)

Rosângela Pereira (Instituto de Nutrição Josué de Castro da Universidade Federal do Rio de Janeiro)

Rosely Sichieri (Instituto de Medicina Social da Universidade Estadual do Rio de Janeiro)

Siléia do Nascimento (Instituto de Medicina Social da Universidade Estadual do Rio de Janeiro)

Taís de Souza Lopes (Instituto de Nutrição Josué de Castro da Universidade Federal do Rio de Janeiro)

Vânia Marins (Fundação Arthur Sá Earp - Petrópolis) 